\title{
OPTIMIZING THE USE OF ANTI VEGF TARGETED THERAPIES IN PATIENTS WITH METASTATIC COLORECTAL CANCER: REVIEW OF LITERATURE
}

\author{
CLAUDIU HOPIRTEAN, VIORICA NAGY
}

\author{
Oncology and Radiotherapy Department, Iuliu Hatieganu University of Medicine \\ and Pharmacy, Cluj-Napoca, Romania
}

\begin{abstract}
Colorectal cancer is one of the most frequent forms of cancer both in men and women, and patients with metastatic disease are now being exposed to an increasing number of therapeutic agents to improve the survival outcomes.

Vascular endothelial growth factor (VEGF) has o key role in the tumor growth and spreading. The approval of 4 agents that target angiogenic pathways in combination with standard chemotherapy improve overall and progression free survival and offer many opportunities to sequencing the treatment in patients with metastatic colorectal cancer $(m C R C)$.

However, the most effective strategy for the use of these agents remains unclear. This article presents an overview of the actual evidence for the use of agents that target angiogenesis in the treatment of $m C R C$.
\end{abstract} VEGF

Keywords: aflibercept, angiogenesis, bevacizumab, ramucirumab, regorafenib,

\section{Introduction}

Colorectal cancer (CRC) is one of the diseases with highest prevalence and mortality and a real public health problem globally, with an incidence over 1.4 million new diagnosed cases and with a mortality of almost 700 thousand deaths each year [1].

For many years there were limited therapeutic options for this pathology, especially for the metastatic disease, comprising either fluoropyrimidine 5-fluorouracil (5-FU) alone, or combinations of fluoropyrimidine with oxaliplatin or irinotecan.

At a growing incidence, new modern therapeutic options to bring an important contribution in terms of improving the results of the treatments was necessary. A qualitative leap has been made with the advent of monoclonal antibodies to ongoing vascular endothelial growth factor (VEGF) and epidermal growth factor receptor (EGFR), which managed to bring an improvement in overall survival

Manuscript received: 30.08.2017

Received in revised form: 16.11.2017

Accepted: 29.11.2017

Address for correspondence: hopirtean.claudiu@gmail.com over 3 years from time of diagnosis [2-4].

Angiogenesis represents one of the most important components of tumor development and metastasis process, and there are two major pathways involved: vascular endothelial growth factor signaling (VEGF) and angiopoietin [5], therefore targeting the process of forming new blood vessels proved to be a good therapeutic option for patients with metastatic colorectal cancer (mCRC), especially as approximately more than half (only $40 \%$ are RAS wild type) of the patients are not eligible for anti EGFR therapies. [6]

There are at least four agents that act at the angiogenesis level in mCRC: bevacizumab - a recombinant humanized monoclonal antibody that blocks angiogenesis by inhibiting vascular endothelial growth factor A (VEGF-A), ramucirumab - a fully human monoclonal antibody that is directed against the vascular endothelial growth factor receptor 2 (VEGFR2), regorafenib - an oral multi-kinase inhibitor which targets oncogenic, stromal and angiogenic receptor tyrosine kinase (RTK) and aflibercept - a recombinant fusion protein, and all of them act like a VEGF-trap, binding the circulating VEGFs (VEGF-A, 
VEGF-B, placental growth factor); in the following we will review major studies according to the line of treatment where they are approved and used.

\section{First line setting}

In a pivotal phase III study, 813 patients with metastatic colorectal cancer, previously untreated, were randomized to get the IFL plus bevacizumab $(5 \mathrm{mg} / \mathrm{kg}$ in 2 weeks) or placebo [7]. There was also a third arm, which enlisted 110 patients who received $5 \mathrm{FU} / \mathrm{LV}$, until an interim analysis that proved that the IFL + Bevacizumab was safe in terms of toxicities, 100 of these patients subsequently being randomized in IFL + placebo arm. Association of Bevacizumab had a better median overall survival (OS) (20.3 vs. 15.6 months) (Table I), a progression free survival (PFS) significantly better (10.6 vs. 6.2 months) (Table I) and a high response rate ( 44.8 vs. 34.8 ), compared to IFL alone. Grade 3 hypertension was the most encountered adverse effect, which was relatively easy to treat with antihypertensives. Bowel perforation was reported in $1.5 \%$ of patients, it was also one case of death. Following these results, the FDA decided to approve Bevacizumab as standard first-line regimen in combination with 5FU-based chemotherapy in patients with mCRC.

NO16966 a randomized, two-arm, non-inferiority phase III comparison of XELOX vs FOLFOX4, which was subsequently amended with further randomization to bevacizumab or placebo, aimed to analyze the efficacy and safety profile of Bevacizumab in combination with first-line chemotherapy based on oxaliplatin. The main objective of this study was PFS. A total of $1401 \mathrm{mCRC}$ patients were randomized after a $2 \times 2$ factorial design, to receive FOLFOX4 or CapOx regimen +/-Bevacizumab [8].

Median PFS was 9.4 months in arm with bevacizumab vs. 8.0 months in the placebo arm $(p=0.0023)$ (Table I) and median OS was 21.3 months in arm with bevacizumab vs. 19.9 months in the placebo arm $(p=0.077)$ (Table I). This study demonstrates noninferiority between FOLFOX4 and CapOx and superiority of associating the chemotherapy with bevacizumab, in terms of PFS. The secondary objective (OS) was not achieved. Toxicity profile was similar to reported data in previous clinical trials.

It was examined if the triple chemotherapy associated with bevacizumab was effective and safe, given as first-line sequence. TRIBE, an open label, multicenter, randomized, phase 3 trial, in 34 oncological centers from Italy, which enlisted patients with nonresectable $\mathrm{mCRC}$, age between 18 and 75 years, ECOG PS of 0-2 (patients aged over 70 years to have an $\mathrm{ECOG}=0$ ), which have not previously received any treatment for metastatic disease [9]. Patients who received adjuvant therapy during the evolution of the disease were enrolled in this study, with the difference that they did not progress in a period of below 12 months from completion of adjuvant treatment. The exclusion criteria were uncontrolled high blood pressure, clotting disorders or major cardiovascular events with 6 months before the start of treatment, which required the administration of a medication.

Patients included in the study were randomized 1:1 in two arms, to receive up to 12 cycles of FOLFOXIRI + Bevacizumab (experimental group) or FOLFIRI + Bevacizumab (control group). After the end of the 12 cycles all patients (both arms) received maintenance treatment with Bevacizumab + fluorouracil, until disease progression.

The main objective of the study was PFS according to RECIST 1.0 [10] or death from any cause. Secondary objectives were OS, response rate, the rate of resectability of metastases and the safety profile in accordance with CTCAE 3.0 [11].

Between 17 July 2008 and 31 May 2011, 508 patients were included in the study, 256 being enrolled in the control arm and 252 in the experimental arm. The two arms were relatively homogeneous in terms of the characteristics of the patients included (age, gender, PS, the location of the primary tumor and metastasis). The analysis is based on statistics from 439 events of 508 patients. The median duration of the follow-up was 32.3 months (24.740.6). Median PFS was 12.1 months in the experimental arm versus 9.7 months in the control arm ( $\mathrm{HR}=0.75,95 \%$ CI $0.62-0.90, p=0.003$ ) (Table I). Median OS was 31.0 months in experimental arm vs. 25.8 months in the control arm $(\mathrm{HR}=0.79,95 \%$ CI $1.00-0.63, \mathrm{p}=0.054)$ statistically insignificant (Table I).

The toxicity profile was acceptable, only $3 \%$ of patients having side effects of grade 3-4, the most common being neutropenia, diarrhea and stomatitis, peripheral neurotoxicity. In conclusion, the combination FOLFOXIRI + Bevacizumab may be a good therapeutic option, with an acceptable safety profile.

\section{Role of maintenance anti VEGF therapy}

The optimal duration of treatment of first line metastatic colorectal cancer has not been clearly established. Also, the role of maintenance therapy was not explored. A lot of treatment strategies were explored including an induction treatment followed by a planned reduction of treatment intensity at least part of the chemotherapy backbone, stop-and-go strategies or chemotherapy holidays (treatment free periods).

AIO 0207 a noninferiority clinical trial compared the combination with fluoropyrimidine and Bevacizumab as standard maintenance treatment vs. Bevacizumab alone vs. observation. After a 24 weeks of induction therapy with fluoropyrimidine, oxaliplatin and bevacizumab patients without disease progression were randomized 1:1:1. The primary end point of this study was time to failure of the strategy, defined as time to second progression after maintenance strategy, death or initiations of another drug sequence [12].

Between Sep. 17, 2009 and Feb. 21, 2013, 837 patients were enrolled and a number of 472 were randomized (158 to receive fluoropyrimidine + Bevacizumab, 156 to 


\section{Oncology}

receive Bevacizumab alone and 158 to receive no treatment). Median follow-up after randomization was 17 months. Median time to failure was 6.9 months for fluoropyrimidine + Bevacizumab combination (95\% CI 6.1-8.5), 6.1 months ((95\% CI 5.3-7.4) for Bevacizumab alone and 6.4 months (95\% CI 4.8-7.6) for the no treatment group $(\mathrm{p}=0.53)$. Time to first progression was 6.2 months for the combination maintenance therapy, 4.8 months in the Bevacizumab alone arm and 3.8 months in no treatment arm $(\mathrm{p}<.001$ for observation vs. bevacizumab or combination therapy). There are no significant differences in overall survival (23.8 months for combination, 26.2 months for bevacizumab alone and 23.1 in no treatment arm) (Table I).

CAIRO3 study aimed to evaluate the effectiveness of maintenance treatment with capecitabine + Bevacizumab vs. observation. This is an open label, randomized, multicenter phase 3 trial, developed in 64 oncologic centers from the Netherlands. Were included patients aged over 18 years who have not previously been treated for metastatic disease, who had at least stable disease after 6 cycles of induction type CAPOX + Bevacizumab, ECOG 0-1, renal and hepatic normal functions [13].

Patients were randomized $1: 1$, in the two arms (with maintenance arm and observational arm). Patients were evaluated at every 9 weeks and when the first disease progressions, commonly referred to as PFS1, patients were reintroduced on induction treatment (CAPOX-B), until the second progression (PFS2) which is the primary objective of this study. The secondary aims of this trial were time to first progression (PFS1), OS, quality of life, overall response rate and toxicity profile.

Between 30 May 2007 and 15 October 2012, a total of 558 patients were randomized 1:1, observational and in the treatment group, 279 patients in each arm. One of the patients in the maintenance arm withdrew the informed consent before the onset of the study. The two arms were well balanced in terms of baseline characteristics (age, gender, status, response to induction therapy, the location of the primary tumor). The median duration of the follow-up was 48 months (36-57). Statistics was conducted when 468 (84\%) of the total of 557 died (239 from 229 observational arms from the arm of maintenance). Median duration to first progression (PFS1) was 8.5 months (95\% CI 6.9-10.2) and the duration up to the second progression (PFS2) was 11.8 months (95\% CI 10.2-13.3) maintenance treatment group, statistically significant better than observational arm (PFS1 4.1 months, and PFS2 10.5 months) (Table I).

The treatment was well tolerated, even though a total of $64(23 \%)$ patients developed hand-foot syndrome during maintenance treatment. The results have proven that maintenance treatment is effective without compromising the quality of life.

\section{Anti VEGF therapy beyond progression}

The ML18147 prospective, randomized openlabel, phase III trial, was developed in 220 centers in 15 countries. Were considered eligible patients with the age $>18$ years, with confirmed mCRC, measurable disease in accordance with RECIST [10] criteria, ECOG PS 0-2, previously treated with bevacizumab + chemotherapy based on fluoropyrimidine and oxaliplatin or irinotecan and which were not candidates to metastasectomy [14]. Patients included in the study with a 1:1 randomization in two arms, have received chemotherapy based on fluoropyrimidine + oxaliplatin or irinotecan with or without bevacizumab in the dose of $2.5 \mathrm{mg} / \mathrm{kg} /$ week associated, in accordance with the chemotherapy regimen preferred by the investigator $(5 \mathrm{mg} /$ $\mathrm{kg}$ at 2 weeks or $7.5 \mathrm{mg} / \mathrm{kg}$ body weight to 3 weeks). The treatment has continued until progression of the disease, unacceptable toxicity or patient death or informed consent withdrawn. The main objective was OS and the secondary objective was PFS. Response to treatment has been evaluated in accordance with the RECIST 1.0 [10] criteria. Adverse reactions have been assessed in agreement with the CTCAE 3.0 [11] and dose reduction of bevacizumab was not accepted.

From 1 Feb. 2006 and until 9 June 2010 a number of 820 patients had been randomized in two arms one with bevacizumab + chemotherapy (409 patients) and another with chemotherapy (411 patients). The median OS was 11.2 months in bevacizumab + chemotherapy arm and 9.8 months in chemotherapy alone arm (Table II), the median PFS has been 5.7 months in bevacizumab + chemotherapy arm and 4.1 months in chemotherapy arm (Table II). Adverse events have appeared in 394 (98\%) of patients from the group

Table I. First line setting and maintenance.

\begin{tabular}{|c|c|c|c|c|c|}
\hline Study (vear) & Treatment & Patients & End points (months) & $\mathrm{HR}(95 \% \mathrm{CI})$ & $p$ ralue \\
\hline \multirow[t]{2}{*}{ Hurwitzet al. AVF2107(2004) } & IFL + placebo vs. $\mathbb{F L}+$ Bevacizumab & 813 & OS (15.6 vs. 20.3) & $0.66(0.54-0.81)$ & $<0.001$ \\
\hline & & $411 \mathrm{vs} .402$ & PFS (6.2 vs. 10.6) & $0.54(0.45-0.66)$ & $<0.001$ \\
\hline \multirow[t]{2}{*}{ Saltzet al. NO16966 (2008) } & CapOX or FOLFOX4 +l- Bevacizumab & $1401(1400)$ & PFS (8.0 vs. 9.4) & $0.83(0.72-0.95)$ & 0.0023 \\
\hline & & 701 vs. 699 & OS (19.9 vs. 21.3$)$ & $0.89(0.76-1.03)$ & 0.0769 \\
\hline \multirow[t]{2}{*}{ Loupalis et al. TRIBE (2014) } & FOLFIRI + Bevacizmab vs. FOLFOXIRI+ Bevacizumab & 508 & PFS (9.7 vs. 12.1) & $0.75(0.62-0.90)$ & 0.003 \\
\hline & & $256 \mathrm{vs} .252$ & OS $(25.8 \mathrm{vs} .31 .0)$ & $0.79(0.63-1.00)$ & 0.054 \\
\hline \multirow[t]{2}{*}{ Hegerwischetal ATO0207(2015) } & Fhoropyimidine + Beracizumab us. Bevacizrmab us, no treatment & $837(472)$ & IFS $(6.9 \mathrm{vs}, 6.1 \mathrm{vs}, 64)$ & $1.08(0.85-1.37)$ & 0.53 \\
\hline & & $15 \mathrm{srs} .156 \mathrm{rs} .158$ & OS (23.8v. $26.2 \mathrm{vs} .23 .1)$ & $0.99(0.71-1.38)$ & 0.7 \\
\hline \multirow{2}{*}{ Simkens et al. CAIR03 (2015) } & no maintenance vs. maintenance Capecitabine + Bevacizumab & 558 & PFS2 (10.5 vs. 11.8$)$ & $0.66(0.54-0.79)$ & $<0.0001$ \\
\hline & & 279 vs. 279 & PFS1 (4.1 vs. 8.5) & $0.40(0.33-0.48)$ & $<0.0001$ \\
\hline
\end{tabular}


bevacizumab + chemotherapy and in 403 (99\%) of patients in the group with chemotherapy. Degree 3-5 reactions have appeared in $255(64 \%)$ of patients in the first group and $235(57 \%)$ patients in the second group. There have been reported 11 adverse events of grade 5 in each group. The most frequent adverse events in both groups were neutropenia, diarrhea and asthenia. A number of 63 patients from the group with bevacizumab + chemotherapy and 36 patients from the group with chemotherapy were forced to cut any kind of treatment because of adverse events. The results of this study demonstrate that bevacizumab administration after the disease progression, changing only the layout of chemotherapy, improve outcomes in patients with mCRC, which have been treated in the first line with bevacizumab. Continuation of bevacizumab brings benefits both in terms of OS and PFS, with acceptable toxicity profile.

\section{Second line setting after previous exposure to anti VEGF agents}

A phase III large trial wanted to demonstrate that the combination Aflibercept + FOLFIRI can improve survival in patients with $\mathrm{mCRC}$ that is resistant to or has progressed after treatment with an oxaliplatin-containing regimen with or without bevacizumab. They were included in the study patients with age $>18$ years of age, performance status (PS) $0-2$, which have histological confirmation. Patients who had received irinotecan in the first line were excluded from the study. Eligible patients were randomized 1:1 to receive aflibercept + FOLFIRI (aflibercept arm), or the placebo + FOLFIRI (control arm). The patients were administered a dose of $4 \mathrm{mg} / \mathrm{kg}$ or placebo intravenously + FOLFIRI, at an interval of two weeks until the disease progression or unacceptable toxicity. Adjustment of doses was allowed, and in the case of need for definitive cessation of FOLFIRI the patients continued aflibercept or placebo and also in the case in which the aflibercept had to be off, the patient has continued to receive FOLFIRI. Was not allowed the passage between the two arms of the study [15]. The primary objective of the study was OS.

From November 2007 and March 20101401 patients were identified in 176 centers from 28 countries. 1226 patients had been randomized to receive placebo or aflibercept in combination with FOLFIRI. The characteristics of the patients and the history of the disease was balanced in the two arms. A number of 373 patients were identified who were previously treated with bevacizumab. At the time of the analysis the median follow-up was 22.28 months. The analysis was based on 403 of events in the arm with aflibercept and 460 events in the control arm. Patients in the arm with aflibercept had an OS significantly higher than in the control arm (13.5 months v. 12.06 months, $\mathrm{p}=.0032$ ) (Table II). At the same time, the patients who had been administered to the combination of aflibercept + FOLFIRI had a median PFS superior to placebo + FOLFIRI. (6.9 months v. 4.7 months, $\mathrm{p}<0.0001$ ) (Table II).

Combination aflibercept + FOLFIRI improved the outcomes in patients with mCRC previously treated with oxaliplatin based regimen with an acceptable safety profile.

Given the known results from other studies which have demonstrated that continuation of a anti VEGF agent in the second line treatment was correlated with an improved survival and PFS, the possibility of introducing the Ramucirumab was analyzed as an option for the second line setting. Ramucirumab is a humanized monoclonal antibody of IgG type-1 which targets the extracellular field of the receptor 2 of VEGF. The RAISE study (224 centers in 24 countries), a randomized, double-blind phase 3 trial proposed to assess the efficacy and safety of treatment with ramucirumab + FOLFIRI vs. Placebo + FOLFIRI, after the progression to treatment with Bevacizumab + fluoropyrimidine + oxaliplatin used in the first line setting [16]. The main objective of the study was OS and secondary objectives PFS and the toxicity profile.

Between 14 Dec. 2010 and 23 Aug. 2013 a number of 1072 patients were enrolled in a study ( 889 of them have received treatment for the first line with Bevacizumab for at least 3 months) and randomized 1:1 by 536 in each arm. The two arms were relatively balanced as regards the characteristics of the patients (age, gender, ethnicity, PS, the number of metastatic sites, the location of the primary tumor). When performing statistical analysis, 769 (72\%) of the patients were dead, 372 in the arm with ramucirumab and 397 in the arm of a placebo. Median OS was 13.3 (95\%CI 12.4-14.5) months in arm with ramucirumab vs. 11.7 (10.8-12.7) months in the placebo arm (HR 0.844, 95\%CI 0.730-0.976, $\mathrm{p}=0.0219$ ) (Table II). The median PFS was superior in the ramucirumab arm vs. placebo arm (5.7 months vs. 4.5 months, $\mathrm{p}=0.0005$ ) (Table II). By analyzing the profile of safety with the two treatments, a larger number of patients from the group with ramucirumab were forced to discontinue treatment because of the toxicities (59 vs. 23), but as a whole the two arms had approximately the same profile of toxicity. It can therefore be concluded that the Ramucirumab is a good choice for the treatment of the second line in patients with mCRC.

Table II. Anti VEGF agents beyond progression and second line after previous exposure to anti VEGF agents.

\begin{tabular}{|c|c|c|c|c|c|}
\hline Study (year) & Treatment & Patients & End points (months) & $\mathrm{HR}(95 \% \mathrm{CI})$ & $p$ value \\
\hline \multirow[t]{2}{*}{ Bennouna et al. ML18147 (2013) } & FOLFOX or FOLFRI +- Bevacizumab beyond progression & 820 & OS (9.8vs. 11.2) & $0.83(0.71-0.97)$ & 0.0211 \\
\hline & & 409 vs. 411 & PFS (4.1 vs. 5.7) & $0.67(0.58-0.78)$ & $<0.001$ \\
\hline \multirow[t]{2}{*}{ Van Cutsem et al. VELOLR (2012) } & placebo + FOLFRI vs. FOLFRI + Aflibercept & $1401(1226)$ & OS (12.06vs. 13.5) & $0.81(0.71-0.93)$ & 0.0032 \\
\hline & & 614 vs. 612 & PFS (4.67 vs. 6.90$)$ & $0.75(0.66-0.86)$ & $<0.0001$ \\
\hline \multirow[t]{2}{*}{ Tabernero et al RAISE (2015) } & placebo + FOLFRI vs. FOLFRI + Ramucinumab & 1072 & OS (11.7 vs. 13.3) & $0.84(0.73-0.97)$ & 0.0219 \\
\hline & & 536 vs. 536 & PFS (4.5 vs. 5.7) & $0.79(0.69-0.90)$ & $<0.0005$ \\
\hline
\end{tabular}


Table III. Second line setting and beyond.

\begin{tabular}{|c|c|c|c|c|c|}
\hline Study (year) & Treatment & Patients & End points (morths) & $\mathrm{HR}(95 \% \mathrm{CI})$ & $p$ ralue \\
\hline Giantonio et al E3200 (2007) & Bevacizumab vs. FOLFOX4 vs. FOLFOX4 + Bevacizumab & $829(820)$ & OS (10.2 vs. 10.8 vs. 12.9$)$ & $0.76(\mathrm{NS})$ & 0.0018 \\
\hline & & $243 \mathrm{vs} .291 \mathrm{vs} .286$ & PFS (2.7 vs. $4.7 \mathrm{vs.7.3)}$ & 0.75 (NS) & 0.0011 \\
\hline Grothey et al CORRECT (2013) & placebo vs. Regorafenib & 760 & OS $(5.0$ vs. 6.4$)$ & $0.77(0.64-0.94)$ & 0.0052 \\
\hline & & 255 vs. 505 & PFS (1.7 พ5. 1.9) & $0.49(0.42-0.58)$ & $<0.0001$ \\
\hline
\end{tabular}
patients

Second line settings to anti VEGF agents naive

Because not all patients have received in the first line an anti VEGF agent, a phase III study was designed with use of bevacizumab in the second line setting (E3200 clinical trial). Thus, a number of 829 patients bevacizumab naive, who had received in the first line of treatment the combination 5 fluorouracil + irinotecan were randomized in three arms to receive one of the following regimens for the second line setting: FOLFOX4 + bevacizumab, FOLFOX 4 or bevacizumab alone. The dose of bevacizumab was greater than that and in other studies conducted with it [17]. It was observed that the combination FOLFOX 4 + bevacizumab had the best results in terms of OS (12.9 vs. 10.8 vs. 10.2 months) (Table III), PFS (Table III) and the response rate. Bevacizumab alone cannot be used with the lower results than chemotherapy alone in terms of response rate and PFS. As regards the profile of the safety of the treatment arm with bevacizumab has had in addition $14 \%$ accompaniment of grade 3 and 4 (hypertension, hemorrhages, nausea and vomiting).

\section{Beyond the second line}

Regorafenib is a small molecule, a rust inhibitor of tyrosine kinase, involved in the functionality of the normal operation of the cells, in the pathological mechanisms, in oncogenesis, in angiogenesis and in maintaining microclimate of the tumors. RET, VEGFR1, VEGFR2, VEGFR3, kit, PDGFR-alpha, PDGFR-beta, FGFR1, FGFR2 and BRAFV600E are all targets of this molecules. The effectiveness of the regorafenib has been evaluated by phase III study (CORRECT study), which included a number of 760 patients with mCRC resistant to previous treatments. Randomization was done $2: 1$ so that a greater number of patients should receive active treatment, the comparison with the effect of a placebo. Regorafenib in the dose of $160 \mathrm{mg} /$ day was administered in the first three weeks of a cycle of 4 weeks [18]. The main objective of the study was the OS which was significantly better in the arm with Regorafenib in comparison with placebo (6.4 months vs. 5.0 months, $\mathrm{p}=0.0052$ ) (Table III). The secondary end point was PFS (Table III). As it was expected, adverse effects in $93 \%$ of patients were reported with regorafenib, compared to $61 \%$ in group a placebo. The grade 3 and 4 toxicities were: hand-foot syndrome in $17 \%$ of patients, fatigue in $10 \%$, diarrhea in $7 \%$ and hypertension in $7 \%$ of the subjects.

There are some preclinical and clinical findings that favor the use of anti-EGFR agents upfront in patients with $\mathrm{mCRC}$, but there are several limitations before a clear conclusion (incomplete data from CALGB and COMETS trials) [19] and another uncertainty remain the role of anti-EGFR drugs in BRAF mutated patients [20]. Maybe PARADIGM trial will find the optimal strategy in patients with RAS wild type mCRC [21].

On the other hand, there are some predictive factors like primary tumor location that seem to impact the outcomes in patients with mCRC. Patients with RAS wild-type tumors arising from the right colon should receive chemotherapy combined with bevacizumab and patients with RAS wildtype tumors from the left colon should receive chemotherapy combined with anti-EGFR therapy [22].

\section{Conclusions}

Patients with metastatic colorectal cancer are candidates for several lines of treatment, with the development of the new options treatment which have evolved from cytostatic agents administered in the couple, up to biological agents used in nowadays. It is a relevant fact that the association of chemotherapy and targeted therapies have brought improvement to the overall survival in the case of these patients. However, no one can say that a certain sequence therapeutic efficacy is higher, Bevacizumab can be managed alongside chemotherapy both in the first line of treatment and in the second line. Moreover, Bevacizumab can be used in the treatment of maintenance and even associated with the triple chemotherapy. Other agents which are targeting angiogenesis (aflibercept, ramucirumab, regorafenib) have been assessed only after the failure of the first line of treatment, but the results obtained are promising. As regards the safety profile, these treatments are different from standard chemotherapy, but grade 3 and 4 adverse events are generally controllable, and only in rare situations in was discontinuation of the treatment necessary.

\section{References}

1. Torre LA, Bray F, Siegel RL, Ferlay J, Lortet-Tieulent J, Jemal A. Global cancer statistics, 2012. CA Cancer J Clin. 2015;65(2):87-108.

2. Van Cutsem E, Köhne CH, Láng I, Folprecht G, Nowacki MP, Cascinu S, et al. Cetuximab plus irinotecan, fluorouracil, and leucovorin as first-line treatment for metastatic colorectal cancer: updated analysis of overall survival according to tumor KRAS and BRAF mutation status. J Clin Oncol. 2011;29(15):2011-2019. 3. Heinemann V, von Weikersthal LF, Decker T, Kiani A, Vehling- 
Kaiser U, Al-Batran SE, et al. FOLFIRI plus cetuximab versus FOLFIRI plus bevacizumab as first-line treatment for patients with metastatic colorectal cancer (FIRE-3): a randomised, openlabel, phase 3 trial. Lancet Oncol. 2014;15(10):1065-1075.

4. Schwartzberg LS, Rivera F, Karthaus M, Fasola G, Canon JL, Hecht JR, et al. PEAK: a randomized, multicenter phase II study of panitumumab plus modified fluorouracil, leucovorin, and oxaliplatin (mFOLFOX6) or bevacizumab plus mFOLFOX6 in patients with previously untreated, unresectable, wildtype KRAS exon 2 metastatic colorectal cancer. J Clin Oncol. 2014;32(21):2240-2247.

5. Warren RS, Yuan H, Matli MR, Gillett NA, Ferrara N. Regulation by vascular endothelial growth factor of human colon cancer tumorigenesis in a mouse model of experimental liver metastasis. J Clin Invest. 1995;95(4):1789-1797.

6. Yancopoulos GD, Davis S, Gale NW, Rudge JS, Wiegand SJ, Holash J. Vascular-specific growth factors and blood vessel formation. Nature. 2000;407(6801):242-248.

7. Hurwitz H, Fehrenbacher L, Novotny W, Cartwright T, Hainsworth J, Heim W, et al. Bevacizumab plus irinotecan, fluorouracil, and leucovorin for metastatic colorectal cancer. N Engl J Med. 2004;350(23):2335-2342.

8. Saltz LB, Clarke S, Díaz-Rubio E, Scheithauer W, Figer A, Wong R, et al. Bevacizumab in combination with oxaliplatin-based chemotherapy as first-line therapy in metastatic colorectal cancer: a randomized phase III study. J Clin Oncol. 2008;26(12):20132019.

9. Loupakis F, Cremolini C, Masi G, Lonardi S, Zagonel $\mathrm{V}$, Salvatore L, et al. Initial therapy with FOLFOXIRI and bevacizumab for metastatic colorectal cancer. N Engl J Med. 2014;371(17):1609-1618.

10. Therasse P, Arbuck SG, Eisenhauer EA, Wanders J, Kaplan RS, Rubinstein L, et al. New guidelines to evaluate the response to treatment in solid tumors. European Organization for Research and Treatment of Cancer, National Cancer Institute of the United States, National Cancer Institute of Canada. J Natl Cancer Inst. 2000;92:205-216.

11. Common Terminology Criteria for Adverse Events v3.0 (CTCAE). Bethesda, MD: Cancer Therapy Evaluation Program, 2006. Available from: https://ctep.cancer.gov/ protocoldevelopment/electronic_applications/docs/ctcaev3.pdf 12. Hegewisch-Becker S, Graeven U, Lerchenmüller CA, Killing B, Depenbusch R, Steffens CC, et al.: Maintenance strategies after first-line oxaliplatin plus fluoropyrimidine plus bevacizumab for patients with metastatic colorectal cancer (AIO 0207): a randomised, non-inferiority, open-label, phase 3 trial. Lancet Oncol. 2015;16:1355-1369.

13. Simkens LH, van Tinteren H, May A, ten Tije AJ, Creemers GJ, Loosveld OJ, et al. Maintenance treatment with capecitabine and bevacizumab in metastatic colorectal cancer (CAIRO3): a phase 3 randomised controlled trial of the Dutch Colorectal Cancer Group. Lancet. 2015;385(9980):1843-1852.

14. Bennouna J, Sastre J, Arnold D, Österlund P, Greil R, Van Cutsem E, et al. Continuation of bevacizumab after first progression in metastatic colorectal cancer (ML18147): a randomised phase 3 trial. Lancet Oncol. 2013;14(1):29-37.

15. Van Cutsem E, Tabernero J, Lakomy R, Prenen H, Prausová $\mathrm{J}$, Macarulla T, et al. Addition of aflibercept to fluorouracil, leucovorin, and irinotecan improves survival in a phase III randomized trial in patients with metastatic colorectal cancer previously treated with an oxaliplatin-based regimen. J Clin Oncol. 2012;30(28):3499-3506.

16. Tabernero J, Yoshino T, Cohn AL, Obermannova R, Bodoky $\mathrm{G}$, Garcia-Carbonero R, et al. Ramucirumab versus placebo in combination with second-line FOLFIRI in patients with metastatic colorectal carcinoma that progressed during or after first-line therapy with bevacizumab, oxaliplatin, and a fluoropyrimidine (RAISE): a randomised, double-blind, multicentre, phase 3 study. Lancet Oncol. 2015;16(5):499-508.

17. Giantonio BJ, Catalano PJ, Meropol NJ, O'Dwyer PJ, Mitchell EP, Alberts SR, et al. Bevacizumab in combination with oxaliplatin, fluorouracil, and leucovorin (FOLFOX4) for previously treated metastatic colorectal cancer: results from the Eastern Cooperative Oncology Group Study E3200. J Clin Oncol. 2007;25(12):1539-1544.

18. Grothey A, Van Cutsem E, Sobrero A, Siena S, Falcone A, Ychou M, et al. Regorafenib monotherapy for previously treated metastatic colorectal cancer (CORRECT): an international, multicentre, randomised, placebo-controlled, phase 3 trial. Lancet. 2013;381(9863):303-312.

19. Zaniboni A, Formica V. The Best. First. Anti-EGFR before anti-VEGF, in the first-line treatment of RAS wild-type metastatic colorectal cancer: from bench to bedside. Cancer Chemother Pharmacol. 2016;78:233-244.

20. Rowland A, Dias MM, Wiese MD, Kichenadasse G, McKinnon RA, Karapetis CS, et al. Meta-analysis of BRAF mutation as a predictive biomarker of benefit from anti-EGFR monoclonal antibody therapy for RAS wild-type metastatic colorectal cancer. Br J Cancer. 2015;112(12):1888-1894.

21. Yoshino T, Uetake H, Tsuchihara K, Shitara K, Yamazaki K, Oki E, et al. PARADIGM study: A multicenter, randomized, phase III study of 5-fluorouracil, leucovorin, and oxaliplatin (mFOLFOX6) plus panitumumab or bevacizumab as firstline treatment in patients with RAS (KRAS/NRAS) wild-type metastatic colorectal cancer. J Clin Oncol. 2016; 34, (suppl 4S; abstr TPS776).

22. Mitchell PA, Sanoff HK Anti-EGFR and Anti-VEGF Agents in First-Line Therapy for Advanced Colorectal Cancer. Current Colorectal Cancer Reports. 2017;13:257-263. 